\title{
4 \\ Indicadores socioeconômicos, nutricionais e de percepção de insegurança alimentar e nutricional em famílias rurais
}

\author{
Dayane de Castro Morais ${ }^{1}$, Naiara Sperandio², Luiza Veloso Dutra ${ }^{3}$, Sylvia do Carmo \\ Castro Franceschini ${ }^{4}$, Ricardo Henrique Silva Santos ${ }^{5}$ e Silvia Eloiza Priore 6
}

\begin{abstract}
A insegurança alimentar e nutricional é multifacetada, apresentando diversos indicadores para esta situação, como os socioeconômicos, de estado nutricional e de percepção quanto ao acesso aos alimentos. O trabalho objetivou avaliar a insegurança alimentar e nutricional de famílias rurais, segundo indicadores socioeconômicos, nutricionais e de percepção. Para tal avaliação, foram adotados indicadores sociais, econômicos, antropométricos, de consumo alimentar e de percepção, segundo a Escala Brasileira de Insegurança Alimentar (EBIA). Trata-se de estudo transversal com famílias residentes na zona rural de um município da zona da Mata Mineira. Das famílias avaliadas, 49,4\% estavam em insegurança alimentar, pela EBIA. Presença de menor de 18 anos, renda per capita, água tratada, número de moradores e de filhos e recebimento de Bolsa Família associaram-se a (in)segurança alimentar. A pontuação da EBIA correlacionouse positivamente com as variáveis: número de moradores, filhos e adolescentes e negativamente com renda total e per capita. O estado nutricional não se associou à (in)segurança alimentar, pela EBIA, embora a maioria das famílias apresentasse algum integrante com distrofia nutricional. Não houve associação entre consumo alimentar da familia e insegurança alimentar, embora todas as famílias apresentassem consumo inadequado de energia e cálcio, e a maioria para sódio, zinco, fibra, ferro, lipídios e carboidratos. Assim, a segurança alimentar e nutricional deve ser avaliada em nível familiar, por diferentes indicadores, visando à avaliação complementar de suas diferentes vertentes.
\end{abstract}

Palavras-chave: segurança alimentar e nutricional, família, indicadores socioeconômicos, estado nutricional, consumo alimentar.

\section{Socio-economic, nutritional and perception indicators of food and nutrition insecurity in rural households}

Food and nutritional insecurity is multifaceted, presenting several indicators for this situation, such as socioeconomic, nutritional status and perception regarding access to food. The objective was to evaluate the food and nutritional insecurity of rural families, according to socioeconomic, nutritional and perception indicators. For this evaluation, we used social, economic, anthropometric, food consumption and perception indicators, according to the Brazilian Food Insecurity Scale (EBIA). This is a cross-sectional study with families living in the rural area

${ }^{1}$ Doutora em Ciência da Nutrição - Universidade Federal de Viçosa (UFV). Assessora Técnica do Centro Colaborador em Alimentação e Nutrição Escolar (CECANE UFV). Departamento de Nutrição e Saúde. Endereço para correspondência: Avenida PH Holfs, s/n, Campus Universitário, Viçosa, MG. E-mail: dayanecm@yahoo.com.br

2 Universidade Federal do Rio de Janeiro (UFRJ). Curso de Nutrição. E-mail: naiarasperandio@yahoo.com.br

${ }^{3}$ Universidade Federal de Viçosa. Departamento de Nutrição e Saúde. E-mail: luizavdutra@gmail.com

${ }^{4}$ Universidade Federal de Viçosa. Departamento de Fitotecnia. E-mail: rsantos@ufv.br

5 Universidade Federal de Viçosa. Departamento de Nutrição e Saúde. E-mail: sylvia@ufv.br

${ }^{6}$ Universidade Federal de Viçosa. Departamento de Nutrição e Saúde. E-mail: sepriore@ufv.br 
of a municipality in the Mata Mineira area. Of the evaluated families, 49.4\% were food insecure, by EBIA. Presence of children under 18, per capita income, treated water, number of residents and children and receipt of Bolsa Familia were associated with (in) food security. The EBIA score correlated positively with the number of residents, children and adolescents and negatively with total and per capita income. Nutritional status was not associated with (in) food security, by EBIA, although most of the families presented some members with nutritional dystrophy. There was no association between family food consumption and food insecurity, although all families had inadequate energy and calcium intake, and most of them had sodium, zinc, fiber, iron, lipids and carbohydrates. Thus, food and nutritional security must be evaluated at the family level, by different indicators, aiming at the complementary evaluation of its different aspects.

Keywords: food and nutrition security, family, socioeconomic indicators, nutritional status, food consumption.

\section{INTRODUÇÃO}

Segurança alimentar e nutricional é definida pelo acesso permanente a alimentos suficientes e de qualidade, para todas as pessoas, sem comprometer o acesso a outras necessidades básicas e que se realize de forma sustentável[1,2].

O conceito ampliado de segurança alimentar e nutricional, somado a complexidade de questões socioeconômicas, psicológicas e nutricionais, relativas a esta situação, evidenciam a necessidade do uso combinado e complementar de diferentes indicadores que abordam as dimensões alimentar e nutricional da mesma, sobrepondo o âmbito individual e abordando a situação familiar ${ }^{[3,4]}$.

Entre os métodos indiretos de avaliação da (in) segurança alimentar e nutricional tem-se os indicadores socioeconômicos, como renda familiar, características do morador de referência do domicílio e acesso aos bens e serviços ${ }^{[5-8]}$; e os nutricionais, com avaliações de medidas antropométricas e dietéticas $[3,9,10]$.

Já como indicador direto de (in)segurança alimentar tem-se a Escala Brasileira de Insegurança Alimentar (EBIA), validada para a população urbana e rural do país. Esse instrumento avalia primeiramente a preocupação do entrevistado em relação à falta de alimento, em seguida a qualidade da alimentação da família e a deficiência em termos quantitativos, primeiro para adultos e depois para crianças, classificando a família em segurança alimentar, insegurança alimentar leve, moderada e grave ${ }^{[3]}$.
Em relação ao risco de insegurança alimentar, a população rural tem sido apontada como mais vulnerável a esta situação em função da menor disponibilidade de recursos socioeconômicos, com consequente monotonia da dieta ${ }^{[11-13]}$.

No Brasil a pesquisa sobre segurança alimentar e nutricional ainda apresenta desafios a serem enfrentados, como a busca por metodologias que englobem a natureza multidimensional desta situação, em seus diferentes níveis de determinação, ou seja, desde o macro até o nível familiar e individual[7]. O presente estudo objetivou avaliar a (in)segurança alimentar e nutricional de familias residentes em zona rural, segundo indicadores socioeconômicos, nutricionais e de percepção.

\section{METODOLOGIA}

\section{Características do estudo}

Trata-se de estudo transversal, com famílias residentes na zona rural de um município da zona da Mata Mineira. Foram incluídas famílias residentes na zona rural do município, desde que todos os integrantes aceitassem participar do estudo.

\section{Cálculo amostral}

Utilizou-se prevalência de 25,5\% de insegurança alimentar em Minas Gerais, detectado pela EBIA[14], erro máximo de $5 \%$, acréscimo de $5 \%$ para possíveis perdas e de $10 \%$ para controle de fatores de confusão, e valor médio de 3,6 indivíduos por domicílio[14], já que a EBIA deve ser aplicada por domicílio, o que resultou em amostra de 78 domicílios. 
O sorteio das famílias se deu a partir do cadastro de agricultores familiares da Empresa de Assistência Técnica e Extensão Rural - EMATER do município, respeitando a proporcionalidade de famílias por comunidade rural. Utilizou-se para cálculo amostral o programa STATCALC do EPI-INFO, versão 6.04.

\section{Avaliação da (in)segurança alimentar e nutricional}

Para avaliação da situação de (in) segurança alimentar e nutricional utilizou-se indicadores socioeconômicos, antropométricos, consumo alimentar, e de percepção (EBIA) (Figura 1).

Figura 1. Indicadores socioeconômicos, nutricionais e de percepção utilizados para avaliação da (in)segurança alimentar e nutricional, em famílias residentes na zona rural

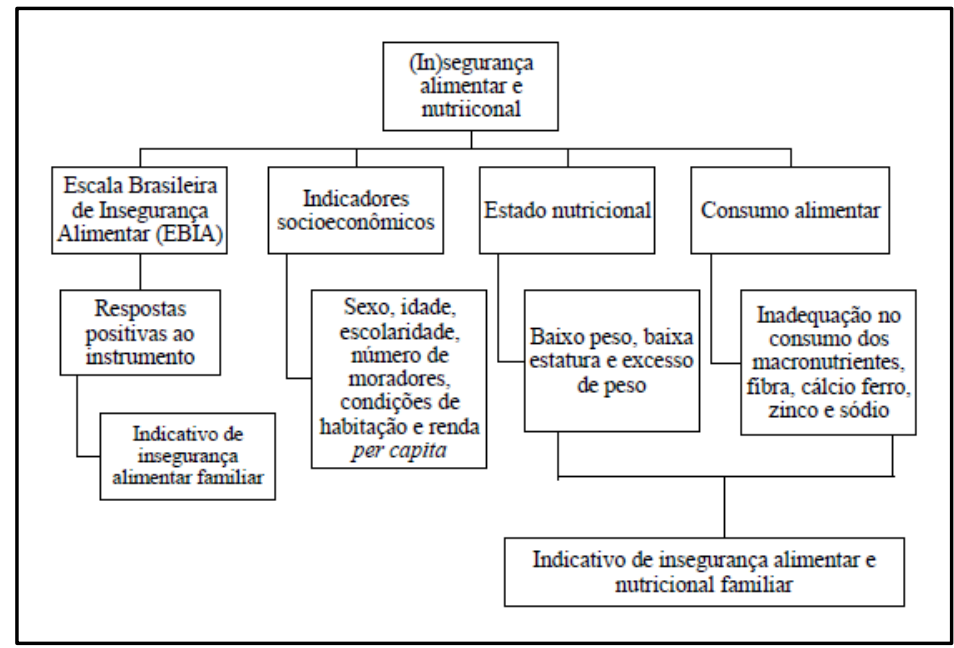

\section{Escala de percepção}

A investigação direta de insegurança alimentar das famílias se deu por aplicação da EBIA ao responsável pela dinâmica alimentar do domicílio, classificando o mesmo em situação de segurança alimentar quando todas as respostas foram negativas. Em domicílios com menores de 18 anos, aplicou-se as 14 questões da escala, classificando o mesmo em insegurança alimentar leve (até cinco respostas afirmativas), moderada (de seis a nove) e grave (acima de dez respostas afirmativas). Nos demais domicílios foram aplicados somente as oito primeiras questões, classificando os mesmos em insegurança alimentar leve (até três respostas afirmativas), moderada (de quatro a seis) e grave (acima de sete respostas afirmativas) ${ }^{[3,15]}$.

\section{Indicadores socioeconômicos}

Os fatores socioeconômicos pesquisados foram sexo, idade, escolaridade, número de moradores, condições de habitação, segundo classificação da Pesquisa sobre Padrões de Vida - PPV[16] e renda per capita, computada somando à renda total da família o valor estimado dos alimentos produzidos para autoconsumo (convertido em valores vigentes no varejo local) ${ }^{[17]}$, com intuito de não subestimar a renda na zona rural[3]. Para classificação da situação de pobreza considerou-se renda per capita inferior a $1 / 2$ salário mínimo e para extrema pobreza renda per capita inferior a $1 / 4$ de salário mínimo ${ }^{[18]}$.

\section{Indicadores nutricionais}

\section{Estado nutricional}

As medidas de peso e estatura de todos os integrantes da família foram aferidas, adotando-se equipamentos específicos para coleta em campo, seguindo técnicas padronizadas. Para classificar o estado nutricional das crianças e adolescentes, foram calculados, segundo sexo, os índices Estatura/Idade e 
IMC/Idade em Z escore; para adultos e idosos IMC; e para gestantes IMC/semana gestacional[19].

Avaliou-se estatura dos adultos com o intuito de verificar presença de desnutrição pregressa também nesta faixa etária, já que este índice é considerado indicador social relacionado ao déficit nutricional cumulativo[2]. Utilizou-se curva da $\mathrm{WHO}^{[21]}$ e ponto de corte para baixa estatura de $<-2$ z. escore aos 19 anos, já que no fim da adolescência o indivíduo já tem seu crescimento finalizado, sendo a baixa estatura correspondente a valores inferiores a 161,9 cm em homens e 150,1 cm em mulheres.

Famílias foram classificadas como em situação de insegurança alimentar e nutricional, pelo estado nutricional, quando pelo menos um de seus integrantes apresentava baixo peso, baixa estatura ou excesso de peso (sobrepeso e/ou obesidade).

\section{Consumo alimentar}

Informações sobre consumo alimentar foram obtidas por um recordatório de ingestão habitual aplicado a todos os indivíduos, sendo os menores de 12 anos e idosos acompanhados por responsável, visando reduzir erros relativos à memória. Questionou-se o que cada indivíduo costuma ingerir por refeição, além do tipo e modo de preparo do alimento, quantidade e marca comercial.

Dados do recordatório alimentar foram analisados no software Diet Pro versão 5.i. Avaliou-se adequação de energia, carboidratos, lipídios ${ }^{[22]}$, gordura saturada ${ }^{[23]}$, proteína, fibras, cálcio, ferro, zinco e sódio[22,24,25].

Considerou-se a família com consumo adequado para energia quando todos os integrantes atingiram às necessidades, segundo Estimated Energy Requeriments (EER) e inadequado quando presença de pelo menos um integrante com consumo abaixo ou acima das necessidades ${ }^{[22]}$. As familias apresentaram consumo adequado para carboidratos e lipídios quando todos os integrantes atingiram às recomendações, e inadequado quando presença de pelo menos um integrante com consumo abaixo ou acima das recomendações, segundo Acceptable Macronutrient Distribuition Range (AMDR) ${ }^{[22]}$. O consumo adequado de proteína na família foi determinado quando todos os integrantes atingiram às recomendações, segundo Dietary Reference Intakes (DRI), para idade, sexo e estado fisiológico e inadequado quando presença de pelo menos um integrante com consumo abaixo das recomendações ${ }^{[22]}$.

As famílias foram consideradas com consumo adequado para gordura saturada quando a totalidade dos integrantes atingiram as recomendações, segundo Sociedade Brasileira de Cardiologia e inadequado quando presença de pelo menos um integrante com consumo acima das recomendações ${ }^{[23]}$. Ao avaliar o consumo da família para fibra, ferro, cálcio, zinco e sódio considerou-se consumo adequado quando todos os integrantes atingiram às recomendações, segundo Dietary Reference Intakes (DRI), para idade, sexo e estado fisiológico e inadequado quando presença de pelo menos um integrante com consumo abaixo das recomendações de fibra, ferro, cálcio e zinco e acima das recomendações de sódio. Considerou-se adequação de sódio e fibra segundo Adequate Intake $(A I)$ e de demais micronutrientes segundo Estimated Average Requirements (EAR).

A presença de inadequação de consumo de energia, macro ou micronutrientes analisados, em pelo menos um membro da família classificou mesma em insegurança alimentar e nutricional.

\section{Aspectos éticos}

Este trabalho foi aprovado pelo Comitê de Ética em Pesquisa com Seres Humanos da Universidade Federal de Viçosa, MG (Registro 241.906/2013). A participação no estudo se deu mediante assinatura do Termo de Consentimento Livre e Esclarecido (TCLE) por todos os membros das famílias ou por seus responsáveis, quando menores de 18 anos. Todas as famílias receberam retorno dos resultados, juntamente com orientações nutricionais específicas de cada situação encontrada.

\section{Análise estatística}

O banco de dados foi validado no EPI-INFO, versão 6.04. Análise dos dados foi realizada no software SPSS versão 20.0. O nível de significância adotado foi menor que 0,05 . Após elaboração de análise estatística descritiva dos dados, foram verificadas associações entre as variáveis categóricas (in)segurança alimentar 
(segundo EBIA) e variáveis socioeconômicas e nutricionais pelo teste de qui-quadrado de Pearson e calculou-se odds ratio. Para as variáveis numéricas foram calculadas correlações de Pearson e Spearman para correlacionar indicadores socioeconômicos e nutricionais com a segurança alimentar, segundo EBIA. Utilizou-se teste de Kappa para análise de concordância entre indicadores de (in)segurança alimentar.

\section{RESULTADOS}

\section{Escala de percepção}

Das familias avaliadas $(\mathrm{n}=79), 49,4 \%(\mathrm{n}=39)$ encontravam-se em insegurança alimentar, segundo
EBIA, estando 87,2\% $(n=34)$ em insegurança alimentar leve e as demais em moderada $(n=4)$ e grave $(n=1)$.

\section{Indicadores socioeconômicos}

Presença de menor de 18 anos, adolescente e menor renda per capita foram as variáveis socioeconômicas da família que condicionaram maior chance de insegurança alimentar domiciliar, segundo EBIA. Já a presença de tratamento de água para consumo (filtração), menor número de moradores, menor número de filhos e recebimento de benefício do Programa Bolsa Família estão relacionadas à situação de segurança alimentar, ou seja, estas características demonstraram menor chance de insegurança alimentar familiar (Tabela 1).

Tabela 1. Variáveis socioeconômicas e situação de (in)segurança alimentar, detectada pela Escala Brasileira de Insegurança Alimentar (EBIA) em famílias residentes de zona rural

\begin{tabular}{|c|c|c|c|}
\hline \multirow[t]{2}{*}{ Variável socioeconômica } & $\begin{array}{c}\text { Segurança alimentar } \\
(\mathrm{n}=40)\end{array}$ & $\begin{array}{c}\text { Insegurança alimentar } \\
(\mathrm{n}=39)\end{array}$ & \multirow{2}{*}{$\begin{array}{l}\text { Odds Ratio* } \\
\text { (IC 95\%) }\end{array}$} \\
\hline & $\mathrm{n}(\%)$ & $\mathrm{n}(\%)$ & \\
\hline Presença $<18$ anos $^{1}$ & & & $3,00(1,19-7,51)$ \\
\hline Sim & $16(40,0)$ & $26(66,7)$ & \\
\hline Não & $24(60,0)$ & $13(33,3)$ & \\
\hline Presença $<5$ anos & & & $1,53(0,55-4,18)$ \\
\hline Sim & $9(22,5)$ & $12(30,8)$ & \\
\hline Não & $31(77,5)$ & $27(69,2)$ & \\
\hline Presença adolescente ${ }^{2}$ & & & $2,77(1,08-7,07)$ \\
\hline Sim & $11(27,5)$ & $20(51,3)$ & \\
\hline Não & $29(72,5)$ & $19(48,7)$ & \\
\hline Presença idoso ${ }^{3}$ & & & $0,60(0,23-1,51)$ \\
\hline $\operatorname{Sim}$ & $17(42,5)$ & $12(30,8)$ & \\
\hline Não & $23(57,5)$ & $27(69,2)$ & \\
\hline Número de moradores & & & $0,33(0,13-0,83)$ \\
\hline $1 \mid-4$ & $28(70,0)$ & $17(43,6)$ & \\
\hline $4|-| 5$ & $11(27,5)$ & $19(48,7)$ & \\
\hline$\geq 6$ & $1(2,5)$ & $3(7,7)$ & \\
\hline Número de filhos & & & $0,27(0,08-0,85)$ \\
\hline 0 & $14(35,0)$ & $5(12,8)$ & \\
\hline $1 \mid-4$ & $25(62,5)$ & $31(79,5)$ & \\
\hline$\geq 4$ & $1(2,5)$ & $3(7,7)$ & \\
\hline Tratamento de água & & & $0,28(0,09-0,90)$ \\
\hline Sim & $35(87,5)$ & $26(66,7)$ & \\
\hline Não & $5(12,5)$ & $13(33,3)$ & \\
\hline Recebe Bolsa Familia & & & $0,29(0,10-0,79)$ \\
\hline Sim & $32(80,0)$ & $21(53,8)$ & \\
\hline Não & $8(20,0)$ & $18(46,2)$ & \\
\hline Renda per capita $(\mathrm{SM})$ & & & $3,50(1,34-9,07)$ \\
\hline$<1 / 4$ & $0(0,0)$ & $7(17,9)$ & \\
\hline $1 / 4 \mid-<1 / 2$ & $10(25,0)$ & $14(35,9)$ & \\
\hline $1 / 2 \mid-<1$ & $16(40,0)$ & $14(35,9)$ & \\
\hline$\geq 1$ & $14(35,0)$ & $4(10,3)$ & \\
\hline Escolaridade morador de referência (em anos) & & & $2,20(0,76-6,30)$ \\
\hline$\leq 4$ & $31(80,0)$ & $27(69,3)$ & \\
\hline$>\overline{4}$ & $8(20,0)$ & $12(30,7)$ & \\
\hline
\end{tabular}


Considerando a situação de pobreza, observou-se que $8,9 \%(\mathrm{n}=7)$ das familias encontravamse em extrema pobreza, estando todas em situação de insegurança alimentar; e 39,2\% $(n=31)$ das famílias em situação de pobreza, estando a insegurança alimentar presente em $67,7 \% \%$ destas $(p<0,001)$.

A pontuação da EBIA por domicílio correlacionou-se positivamente com número de moradores $(r=0,360)$, filhos $(r=0,331)$ e adolescentes $(r=0,299)$ e negativamente com renda total $(r=-0,236)$, renda per capita $(r=-0,412)$, e pontuação da Pesquisa sobre Padrões de Vida (PPV-IBGE, 1998) $(r=-0,371)$ $(p<0,05)$. Todas as famílias foram classificadas na categoria $\mathrm{B}$, segundo $\mathrm{PPV}$, com pontuação variando de 34 a 48 pontos.

\section{Indicadores nutricionais}

\section{Estado nutricional}

A (in)segurança alimentar não se associou à presença de baixo peso, baixa estatura, sobrepeso e obesidade na família (Tabela 2). Ressalta-se que nenhum domićlio em insegurança alimentar moderada e grave apresentou morador com baixo peso e que a presença de pelo menos uma distrofia nutricional na familia esteve presente em $88,2 \%(n=30)$ dos domicilios em insegurança alimentar leve. Não foi captada correlação entre número de indivíduos com baixo peso, baixa estatura, sobrepeso ou obesidade na familia com a (in)segurança alimentar.

O estado nutricional materno não se associou com o de crianças e adolescentes, embora 88,9\% ( $\mathrm{n}=9)$ das crianças e 44,4\% $(n=4)$ dos adolescentes com baixo ou excesso de peso tivessem mães com excesso de peso.

Tabela 2. Estado nutricional na família e situação de (in)segurança alimentar, detectada pela Escala Brasileira de Insegurança Alimentar (EBIA) em famílias residentes de zona rural

\begin{tabular}{|c|c|c|c|c|}
\hline \multirow{2}{*}{ Estado nutricional na família } & \multicolumn{2}{|c|}{ Segurança Alimentar $(n=40)$} & \multicolumn{2}{|c|}{ Insegurança Alimentar $(n=39)$} \\
\hline & $\mathrm{n}$ & $\%$ & n & $\%$ \\
\hline Baixo peso na família ${ }^{1}$ & 10 & 25,0 & 9 & 23,1 \\
\hline Baixa estatura na família ${ }^{2}$ & 11 & 27,5 & 14 & 35,9 \\
\hline Sobrepeso na família ${ }^{3}$ & 21 & 52,5 & 22 & 56,4 \\
\hline Obesidade na família ${ }^{4}$ & 9 & 22,5 & 11 & 28,2 \\
\hline Distrofia na família ${ }^{5}$ & 30 & 75,0 & 33 & 84,6 \\
\hline
\end{tabular}

sobrepeso, pelo IMC; ${ }^{4}$ Presença de pelo menos um integrante da família com obesidade, pelo IMC; 5 Presença de pelo menos um integrante da família com baixo peso, baixa estatura ou excesso de peso (sobrepeso/obesidade); ${ }^{*}$ Teste de qui-quadrado $(p>0,05)$.

\section{Consumo alimentar}

Não houve associação entre consumo alimentar da família e (in)segurança alimentar. Observase que nenhuma família apresentou consumo adequado de todos os integrantes para energia, gordura saturada e cálcio, independente da situação de (in)segurança alimentar.

A maior presença de adequação de consumo de macro e micronutrientes foi observada entre familias em situação de segurança alimentar, enquanto de fibra foi entre famílias em insegurança alimentar (Tabela 3).

Observou-se consumo de gordura de porco em $54,4 \%(n=43)$ das famílias, sendo as famílias em insegurança alimentar responsáveis por 55,8\% ( $\mathrm{n}=24)$ deste consumo. Já o consumo de café adoçado esteve presente em 98,7\% $(n=78)$ das famílias, sendo este consumo distribuído igualmente entre domićlios considerados seguros e inseguros $(50 \%)$. O consumo médio de café adoçado nos domicílios não se correlacionou à pontuação da EBIA. 
Tabela 3. Ingestão de energia e macronutrientes na família e situação de (in)segurança alimentar, detectada pela Escala Brasileira de Insegurança Alimentar (EBIA), em famílias residentes de zona rural

\begin{tabular}{|c|c|c|c|c|}
\hline \multirow{2}{*}{$\begin{array}{l}\text { Consumo alimentar } \\
\text { inadequado }\end{array}$} & \multicolumn{2}{|c|}{ Segurança Alimentar $(n=40)$} & \multicolumn{2}{|c|}{ Insegurança Alimentar $(n=39)$} \\
\hline & n & $\%$ & $\mathrm{n}$ & $\%$ \\
\hline Energia 1 & 40 & 50,6 & 39 & 100,0 \\
\hline Carboidratos $^{2}$ & 21 & 47,7 & 23 & 59,0 \\
\hline Proteína $/ \mathrm{kg}$ de peso $^{3}$ & 11 & 39,3 & 17 & 43,6 \\
\hline Lipídio $^{2}$ & 35 & 48,6 & 37 & 94,9 \\
\hline Gordura Saturada ${ }^{4}$ & 40 & 50,6 & 39 & 100,0 \\
\hline Fibra $^{5}$ & 34 & 51,5 & 32 & 82,1 \\
\hline Ferro $^{5}$ & 25 & 50,0 & 25 & 64,1 \\
\hline Cálcio ${ }^{5}$ & 40 & 50,6 & 39 & 100,0 \\
\hline Zinco $^{5}$ & 37 & 50,0 & 37 & 94,9 \\
\hline Sódio ${ }^{5}$ & 37 & 48,7 & 39 & 100,0 \\
\hline
\end{tabular}

${ }^{1}$ Família com consumo inadequado para energia quando presença de pelo menos um integrante com consumo abaixo ou acima das necessidades (Estimated Eneryy Requeriments - EER); ${ }^{2}$ Família com consumo inadequado para carboidrato ou lipídios quando presença de pelo menos um integrante com consumo abaixo ou acima das recomendações (Acceptable Macronutrient Distribuition Range - AMDR); ${ }^{3}$ Família com consumo inadequado para proteína quando presença de pelo menos um integrante com consumo abaixo das recomendações, segundo idade, sexo e estado fisiológico (Dietary Reference Intakes - DRI); 4 Família com consumo inadequado para gordura saturada quando presença de pelo menos um integrante com consumo acima das recomendações (Sociedade Brasileira de Cardiologia). ${ }^{5}$ Família com consumo inadequado quando presença de pelo menos um integrante com consumo abaixo das recomendações de fibra, ferro, cálcio e zinco e acima das recomendações de sódio, para idade, sexo e estado fisiológico

(Dietary ReferenceIntakes - DRI). Teste de qui-quadrado $(p>0,05)$.

Considerando a situação de insegurança alimentar e nutricional no domicilio, encontrou-se $49,4 \%(n=39)$ de insegurança alimentar pela EBIA, em seus diferentes níveis; 84,8\% (n=67), segundo antropometria, considerando presença de distrofia em pelo menos um dos integrantes da família. Em relação ao consumo alimentar, observou-se que a maioria das famílias estava exposta à insegurança, considerando a inadequação de consumo dos micronutrientes e fibra, bem como dos macronutrientes e de energia (Figura 2).

Figura 2. Situação de insegurança alimentar, segundo Escala Brasileira de Insegurança Alimentar (EBIA), indicadores antropométricos e dietéticos em famílias residentes de zona rural

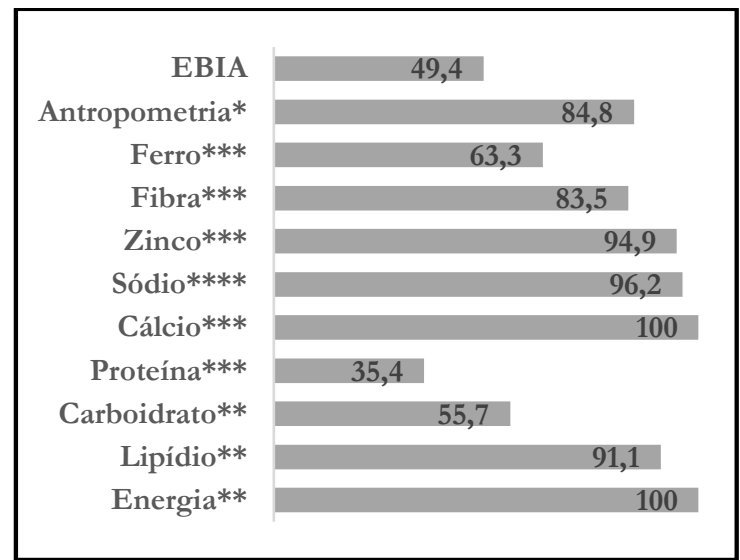

EBIA: Escala Brasileira de Insegurança Alimentar; *Antropometria: família considerada insegura quando presença de pelo menos um integrante com baixo peso, baixa estatura ou excesso de peso; ** Família considerada insegura quando consumo de energia, carboidratos e lipídios abaixo ou acima das recomendações em pelo menos um dos integrantes; *** Família considerada insegura quando consumo de proteína, fibra, ferro, cálcio e zinco abaixo das recomendações em pelo menos um dos integrantes; **** Família considerada insegura quando consumo de sódio ultrapassa as recomendações em pelo menos um dos integrantes. 
A insegurança alimentar detectada pela EBIA não apresentou concordância, segundo teste de Kappa, com a detectada pela presença de baixo peso e baixa estatura na família nem com o consumo abaixo das necessidades de energia para pelo menos um membro da família.

\section{DISCUSSÃO}

A insegurança alimentar nas famílias residentes de zona rural, detectada pela EBIA, apresentou-se superior aos 35,3\% encontrados no Brasil rural e 18,4\% em Minas Gerais ${ }^{[15]}$. Estudos pontuais da situação de insegurança alimentar, em municípios ou regiões brasileiras, encontraram valores entre $40,9 \%$ a $88,5 \%$, com maiores prevalências na zona rural[26].

A distribuição heterogênea da (insegurança alimentar, com discrepâncias entre localidades, indica a necessidade de avaliação de dados municipais, possibilitando assim conhecer as características locais que possam passar despercebidas quando analisadas em unidades estaduais ou regionais, e traçar políticas públicas direcionadas, já que o município é unidade privilegiada para implementação de ações promotoras de segurança alimentar e nutricional[27,28].

Indicadores socioeconômicos, comumente relatados na literatura, que apresentaram relação com insegurança alimentar neste estudo são a presença de menor de 18 anos ${ }^{[2,8]}$; menores rendimentos familiares per capita[5,6,27,29-31]; ausência de água tratada[8,22]; não recebimento de Bolsa Família[8,11]; maior número de moradores[2,5,8] e maior número de filhos ${ }^{[29]}$.

Acredita-se que a associação de insegurança alimentar com os indicadores supracitados decorrem da presença de menor de 18 anos implicar em pais mais jovens e famílias maiores, resultando em maiores gastos, além do fato da infância e adolescência serem especialmente vulneráveis às dimensões alimentar e nutricional da segurança ${ }^{[8]}$; menores valores de renda resultam em restrições econômicas com consequente dificuldade de aquisição e consumo do alimento por um ou mais membros da familia [7,32]; transferência condicionada de renda pelo Bolsa Família implica em aumento de renda na familia, com consequente melhoria de acesso ao alimento[33].
Observou-se também associação da insegurança alimentar com a presença de adolescentes na família, fato este ainda pouco relatado na literatura, uma vez que outros estudos apontam a presença de crianças na família como fator de vulnerabilidade ${ }^{[7,10,27]}$, não questionando a relação entre presença de adolescentes e esta situação.

A correlação encontrada entre maior pontuação da Pesquisa de Padrão de Vida (PPV) e menor pontuação da EBIA pode ser explicada pelo fato da insegurança alimentar estar associada, em outros estudos, com material de construção do domicílio, ausência de água encanada, saneamento básico e coleta de lixo, nas zonas urbana e rural[ $[$.

Insegurança alimentar não se associou à escolaridade do morador de referência da família, de forma distinta do encontrado em outros estudos $[13,27,34,35]$. Sabe-se que escolaridade no meio rural é baixa[13], sendo que neste trabalho $74,7 \%$ dos moradores de referência apresentavam até quatro anos de estudo, indicando homogeneidade da população para essa variável, o que interferiu em seu poder discriminante.

Embora relação estatística ausente entre estado nutricional na família e situação de (in) segurança alimentar, detectada pela EBIA, neste estudo, domićlios em insegurança alimentar, principalmente leve, apresentaram alta prevalência de excesso de peso, além de coexistência de baixo peso e excesso de peso na família. Essa relação entre insegurança alimentar e excesso de peso tem sido explicada por diferentes mecanismos: 1) famílias com restrições econômicas, normalmente em situação de insegurança alimentar, primeiramente substituem os alimentos normalmente consumidos por alimentos mais baratos, sendo estes muitas vezes menos nutritivos e altamente energéticos, levando ao excesso de peso[36; 2) indivíduos acima do peso podem considerar que sua família apresenta insuficiência de alimentos e consequente insegurança alimentar em função da sua visão de grande necessidade de quantidade de alimento para consumo; 3) momentos de compulsão alimentar, decorrentes da ansiedade e incerteza quanto ao acesso ao alimento, podem ocorrer quando o alimento está disponível no domicílio em insegurança alimentar, resultando em excesso de peso ${ }^{[37}$; 4) indivíduos em insegurança alimentar que passam por jejum constante até mesmo no período intrauterino (quando filhos de gestantes desnutridas), 
podem sofrer adaptações metabólicas para poupar energia, resultando em excesso de peso ${ }^{[37]}$.

A coexistência de baixo peso, principalmente em crianças, e excesso de peso na mesma família caracteriza o processo de transição nutricional[38]. Esta transição nutricional, vivenciada pelas famílias brasileiras, pode ser uma das justificativas da não associação entre estado nutricional e situação de insegurança alimentar, nos domićlíos avaliados ${ }^{[2,31]}$.

Ressalta-se que não foi identificada na literatura científica nenhum estudo que relacionasse situação de (in)segurança alimentar com estado nutricional na família. Publicações referentes a este tema abordam estado nutricional individual, em faixas

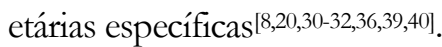

Em relação ao consumo de alimentos observou-se, sem significância, percentual mais elevado de indivíduos que consumiam proteínas e ferro em domicílios seguros, pela EBIA, assim como relatado em outros estudos [5,31,41]. Este fato pode ser justificado pelo preço, geralmente mais elevado, dos alimentos proteicos, que dificultam o acesso das famílias em insegurança alimentar a alimentos fontes ${ }^{[10]}$.

Considerando-se presença de insegurança alimentar e nutricional por indicadores nutricionais obteve-se $84,8 \%$ de famílias inseguras pela antropometria, valor este muito acima do encontrado pela EBIA, indicando que dados antropométricos devem ser considerados na avaliação desta situação já que refletem utilização dos alimentos pelo corpo, sendo uma consequência fisiológica potencial, mas não necessária, da insegurança alimentar e nutricional[4].

Em relação ao consumo de alimentos na família, a insegurança alimentar e nutricional variou de $35,4 \%$ para consumo insuficiente de proteína a $100 \%$ para consumo inadequado de energia e cálcio, apresentando valores de inadequação para os outros nutrientes analisados (carboidrato, lipídio, fibra, ferro, zinco e sódio), característicos de insegurança, superiores ao encontrado pela EBIA. Essas informações relativas ao consumo de alimentos pela família apontam a necessidade de inclusão de dados dietéticos na avaliação da segurança alimentar e nutricional incorporando análise da dimensão nutricional desta situação, em termos quantitativos e qualitativos.
Ressalta-se que a utilização de recordatório de ingestão habitual, embora não validado para investigação do consumo alimentar, tem sido utilizado em populações que apresentam monotonia na ingestão alimentar como em crianças e idosos ${ }^{[42,43,44]}$. O presente estudo avaliou o consumo de residentes na zona rural, geralmente marcado pela ingestão de alimentos tradicionais e baixa variedade, no contexto familiar, justificando assim a utilização deste inquérito alimentar para coleta de dados junto a este público, tendendo a minimizar potenciais vieses de avaliação de consumo alimentar em dias pontuais, como nos demais inquéritos ${ }^{[45]}$.

A discrepância dos resultados de insegurança alimentar (e nutricional) pelos diferentes indicadores decorre da natureza multifacetada da mesma e implica na necessidade de utilização de métodos complementares na avaliação desta situação ${ }^{[3,4]}$, uma vez que a EBIA engloba apenas a dimensão alimentar da insegurança, com foco na percepção dos indivíduos em relação ao acesso aos alimentos[7], e já os indicadores antropométricos e de consumo de alimentos incorporam a dimensão nutricional[46].

A abordagem das distintas dimensões da (in)segurança pelos diferentes indicadores, refletida pelos diferentes resultados de insegurança alimentar e nutricional ressalta a necessidade do uso conjunto de indicadores para avaliação desta situação, uma vez que cada indicador incorpora uma vertente da (in)segurança alimentar e nutricional.

Este estudo é inovador por tratar o aspecto familiar da (in)segurança alimentar e nutricional, considerando todos os membros da família, na zona rural, por diferentes indicadores socioeconômicos e nutricionais, mas apresenta a limitação de ser pontual, impossibilitando definição de causalidade no âmbito da situação de segurança.

\section{CONSIDERAÇÕES FINAIS}

Neste estudo, observou-se relação entre alguns indicadores socioeconômicos e nutricionais e a insegurança alimentar, segundo a EBIA. A insegurança alimentar, avaliada por indicadores nutricionais apresentou, em sua maioria, maiores prevalências do que a detectada pela EBIA, indicando necessidade de inclusão desses indicadores na detecção da insegurança alimentar e nutricional. 
Insegurança alimentar e nutricional e famílias rurais. Morais $e t$ al.

O processo para avaliação da (in)segurança alimentar e nutricional é complexo, requerendo a busca contínua de indicadores viáveis que incorporem as dimensões alimentar e nutricional desta situação de forma mais direta e complementar, revelando assim suas especificidades.

A segurança alimentar e nutricional deve ser avaliada em nível familiar, pois esta situação é multifacetada, afetando primeiramente o domicílio e posteriormente os indivíduos. Por esse motivo, a análise dos fatores associados a (in)segurança alimentar e nutricional intrafamiliar deve ser realizada visando à elaboração de indicadores de estado nutricional e consumo de alimentos da família para a detecção precoce desta problemática.

\section{REFERÊNCIAS}

[1] Brasil. Lei no 11.346, de 15 de setembro de 2006. Dispõe sobre a Criação do Sistema Nacional de Segurança Alimentar e Nutricional. Diário Oficial da União. 18 set 2006.

[2] Hoffmann R. Determinantes da Insegurança Alimentar no Brasil: Análise dos Dados da PNAD de 2004. Segur. Aliment. Nutr. 2008;15(1):49-61.

[3] Segall-Corrêa AM. Insegurança alimentar medida a partir da percepção das pessoas. Estud. Av. 2007;21(60):143-54.

[4] Kepple AW, Segall-Corrêa AM. Conceituando e medindo segurança alimentar e nutricional. Ciênc. Saúde Coletiva. 2011;16:187-99.

[5] Panigassi G, Segall-Corrêa AM, Marin-León L, Pérez-Escamilla R, Maranha LK, Sampaio MFA. Insegurança alimentar intrafamiliar e perfil de consumo de alimentos. Rev. Nutr. 2008;21:135-44.

[6] Vianna RPT, Segall-Corrêa AM. Insegurança alimentar das famílias residentes em municípios do interior do estado da Paraíba, Brasil. Rev. Nutr. 2008;21:111-22.

[7] Gubert MB, Santos LMP. Determinantes da insegurança alimentar no Distrito Federal. Com. Ciências Saúde 2009;2(2):143150 .

[8] Guerra LDS, Espinosa MM, Bezerra ACD, Guimaraes LV, Lima-Lopes MA. Insegurança alimentar em domicílios com adolescentes da Amazônia Legal Brasileira: prevalência e fatores associados. Cad. Saúde Pública. 2013;29(2):335-48.

[9] Monteiro CA. A dimensão da pobreza, da desnutrição e da fome no Brasil. Estud. Av. 2003;48:7-20.
[10] Drewnowski A, Specter SE. Poverty and obesity: The role of energy density and energy costs. Am. J. Clin. Nutr. 2004;79(1):6-16.

[11] Segall-Corrêa AM, Marin-Leon L, Helito H, Pérez-Escamilla R, Santos LMP, Paes-Sousa AR. Transferência de renda e segurança alimentar no Brasil: análise dos dados nacionais. Rev. Nutr. 2008;21:39-51.

[12] Instituto Brasileiro de Geografia e Estatísticas. Cidades. Censo 2010. Rio de Janeiro: IBGE; 2010.

[13] Mondini L, Rosa TE, Gubert MB, Sato GS, Benício MHD. Insegurança alimentar e fatores sociodemográficos associados nas áreas urbana e rural do Brasil. Informações Econômicas. 2011;41(2):52-60.

[14] Instituto Brasileiro de Geografia e Estatísticas. Pesquisa Nacional por Amostra de Domicilios (PNAD). Segurança Alimentar: 2009. Rio de Janeiro: IBGE; 2010.

[15] Instituto Brasileiro de Geografia e Estatística. Pesquisa Nacional por Amostra de Domicílios (PNAD). Segurança alimentar: 2013. Rio de Janeiro: IBGE; 2014.

[16] Instituto Brasileiro de Geografia e Estatística. Pesquisa sobre Padrões de Vida: 1996-1997. Rio de Janeiro: IBGE; 1998.

[17] Takagi M, Silva JG, Grossi MD. Pobreza e Fome: em busca de uma metodologia para quantificação do problema no Brasil. Texto para Discussão. Campinas: IE/UNICAMP; 2001.

[18] Maluf RSJ. Definindo segurança alimentar e nutricional. In: Maluf RSJ, organizador. Segurança alimentar e nutricional. Petrópolis: Vozes; 2007. p.17-19.

[19] Brasil. Ministério da Saúde. Secretaria de Atenção à Saúde. Departamento de Atenção Básica. Orientações para a coleta e análise de dados antropométricos em serviços de saúde: Norma Técnica do Sistema de Vigilância Alimentar e Nutricional -SISVAN/Ministério da Saúde, Secretaria de Atenção à Saúde, Departamento de Atenção Básica. Brasília (DF): Ministério da Saúde; 2011.

[20] Oliveira JS, Lira PIC, Andrade SLLS, Sales AC, Maia SR, Batista Filho M. Insegurança alimentar e estado nutricional de crianças de São João do Tigre, no semi-árido do Nordeste. Rev. Bras. Epidemiol. 2009;12(3):413-23.

[21] World Health Organization. Development of a WHO growth reference for school-aged children and adolescents. Bulletin of the World Health Organization 2007. Roma: WHO; 2007. p. 660-667.

[22] Institute of Medicine. In: Dietary References Intake s for energy, carboidrate, fiber, fat, fatty acids, cholesterol, protein and aminoacids. Washington, DC: The National Academy Press; 2002.

[23] Sociedade Brasileira de Cardiologia. I Diretriz sobre o Consumo de Gorduras e Saúde Cardiovascular. São Paulo: SBC; 2013. 
[24] Institute of Medicine. In: Dietary References Intake s for vitamin A, vitamin $\mathrm{K}$, arsenic, boron, chromium, copper, iodine, iron, manganese, molybdenum, nickel, silicon, vanadium and zinc. Washington, DC: The National Academy Press; 2001.

[25] Institute of Medicine. In: Dietary References Intake s for calcium and vitamin D. Washington, DC: The National Academy Press; 2010.

[26] Morais DC, Dutra LV, Franceschini SCC, Priore SE. Insegurança alimentar e indicadores antropométricos, dietéticos e sociais em estudos brasileiros: uma revisão sistemática. Ciênc. Saúde Coletiva. 2014;19(5):1475-88.

[27] Gubert MB, Benicio MHD, Silva JP, Rosa TEC, Santos SM. Use of predictive model for food insecurity estimates in Brazil. Arch. Latinoam. Nutr. 2010;60(2):119-25.

[28] Silva CCS, Oliveira KBB, Alves AS, Neves JA, Modesto CAC, Vianna RPT. Associação entre consumo alimentar e (in)segurança alimentar e nutricional em São José dos Ramos - PB. Braz. J. Food Technol. 2012;15:23-30.

[29] Salles-Costa R, Pereira RA, Vasconcellos MTL, Veiga GV, Marins VMR, Jardim BC, et al. Associação entre fatores socioeconômicos e insegurança alimentar: estudo de base populacional na Região Metropolitana do Rio de Janeiro, Brasil. Rev. Nutr. 2008;21:99-109.

[30] Velásquez-Melendez G, Schlussel MM, Brito AS, Silva AAM, Lopes-Filho J, Kac G. Mild but not light or severe food insecurity is associated with obesity among brazilian women. J. Nutr. 2011;141(5):898-902.

[31] Almeida JA, Santos AS, Nascimento MAO, Oliveira JVC, Silva DG, Mendes-Netto RS. Fatores associados ao risco de insegurança alimentar e nutricional em famílias de assentamentos rurais. Ciênc. Saúde Coletiva. 2017;22(2):479-488.

[32] Oliveira JS, Lira PIC, Maia SR, Sequeira LAS, Amorim RCA, Batista Filho M. Insegurança alimentar e estado nutricional de crianças de Gameleira, zona da mata do Nordeste brasileiro. Rev. Bras. Saúde Matern. Infant. 2010;10(2):237-45.

[33] Lima FEL, Fisberg RM, Uchimura KY, Picheth T. Programa Bolsa-Família: qualidade da dieta de população adulta do município de Curitiba, PR. Rev. Bras. Epidemiol. 2013;16(1):58-67.

[34] Panigassi G, Segall-Corrêa AM, Marin-León L, Pérez-Escamilla R, Sampaio MFA, Maranha LK. Segurança alimentar como indicador de iniquidade: análise de inquérito populacional. Cad. Saúde Pública. 2008;24(10):2376-84.

[35] Anschau FR, Matsuo T, Segall-Corrêa AM. Insegurança alimentar entre beneficiários de programas de transferência de renda. Rev. Nutr. 2012;25(2):177-89.
[36] Kac G, Velasquez-Melendez G, Schlussel MM, Segall-Corrêa AM, Silva AAM, Perez-Escamilla R. Severe food insecurity is associated with obesity among Brazilian adolescent females. Public Health Nutrition. 2012;15(10):1854-60.

[37] Alaimo K, Olson CM, Frongillo EA. Low family income and food insufficiency in relation to overweight in US children: is there a paradox? Arch. Pediatr. Adolesc. Med. 2001;155(10):1161-1167.

[38] Doak CM, Adair LS, Bentley M, Monteiro C, Popkin BM. The dual burden household and the nutrition transition paradox. Int. J. Obes. 2005;29:129-36.

[39] Oliveira JS, Lira PIC, Veras ICL, Maia SR, Lemos MCC, et al. Estado nutricional e insegurança alimentar de adolescentes e adultos em duas localidades de baixo índice de desenvolvimento humano. Rev. Nutr. 2009;22(4):453-65.

[40] Souza NN, Moura MD, Sperandio N, Franceschini SCC, Priore SE. Perfil socioeconômico e insegurança alimentar e nutricional de famílias beneficiárias do Programa Bolsa Família no município de Viçosa, Estado de Minas Gerais, Brasil, em 2011: um estudo epidemiológico transversal. Epidemiol. Serv. Saúde. 2012;21:655-62.

[41] Antunes MM, Sicheri R, Salles-Costa R. Consumo alimentar de crianças menores de três anos residentes em área de alta prevalência de insegurança alimentar domiciliar. Cad. Saúde Pública. 2010;23(4):785-93.

[42] Batista MCR, Priore SE, Rosado LEFPL, Tinôco ALA, Franceschini SCC. Avaliação dietética dos pacientes detectados com hiperglicemia na "Campanha de Detecção de Casos Suspeitos de Diabetes” no Município de Viçosa, MG. Arq. Bras. Endocrinol. Metab. 2006;50(6).

[43] Novaes JF, Franceschini SCC, Priore SE. Hábitos alimentares de crianças eutróficas e com sobrepeso em Viçosa, Minas Gerais, Brasil. Rev. Nutr. 2007;20(6):633-642.

[44] Fernandes DPS. Qualidade da dieta e fatores associados em idosos de Viçosa (MG): um estudo de base populacional [dissertação]. Viçosa: Universidade Federal de Viçosa; 2016.

[45]Venturini CD, Engroff P, Sgnaolin V, Kik RME, Morrone FB, Silva Filho, et al. Consumo de nutrientes em idosos residentes em Porto Alegre (RS), Brasil: um estudo de base populacional. Ciênc. Saúde Coletiva. 2015; 20(12):3701-3711.

[46] Pérez-Escamilla R, Segall-Corrêa AM. Indicadores e medidas de insegurança alimentar. Rev. Nutr. 2008;21:15-26. 